\title{
ON SUMMABILITY OF FOURIER SERIES AT A POINT
}

\author{
G. D. DIKSHIT
}

ABSTRACT. In this paper summability of Fourier series by a regular linear method of summation determined by a triangular matrix, has been studied and various results - some known and some new - on Cesàro and Nörlund summability have been deduced. A convergence criterion has also been obtained.

1. Let $C=\left(c_{n, k}\right), k=0,1,2, \ldots, n$, be a triangular matrix and let

$$
t_{n}=\sum_{k=0}^{n} c_{n, k} s_{k}
$$

where $\left\{s_{k}\right\}$ is a given sequence of numbers. If $t_{n} \rightarrow s$ as $n \rightarrow \infty,\left\{s_{n}\right\}$ is called summable $(C)$ to $s$. In this paper we assume $c_{n, k} \geq 0$ for $k=0,1,2, \ldots, n$, and $\sum_{k=0}^{n} c_{n, k}=1$. Then a necessary and sufficient condition for regularity of the method $(C)$ is

$$
\lim _{n \rightarrow \infty} c_{n, k}=0 \quad \text { for each } \mathrm{k} \text {. }
$$

In the case

$$
c_{n, k}=A_{n-k}^{\alpha-1} / A_{n}^{\alpha}, \quad \alpha \geq 0,
$$

where $\left\{A_{n}^{\alpha-1}\right\}$ is determined by the identity

$$
(1-x)^{-\alpha}=\sum_{0}^{\infty} A_{n}^{\alpha-1} x^{n} \quad(|x|<1),
$$

the method $(C)$ reduces to the well-known Cesàro method $(C, \alpha)$. For

$$
c_{n, k}=p_{n-k} / P_{n}, \quad P_{n}=p_{0}=p_{1}+\cdots+p_{n}>0,
$$

the method $(C)$ reduces to the Nörlund method $(N, p)$. In the case $p_{n}=1 /(n+1)$, the Nörlund method $(N, 1 /(n+1))$ is also known as the harmonic method.

Let $f$ be a Lebesgue integrable periodic function with period $2 \pi$ and let

$$
f(x) \sim \frac{1}{2} a_{0}+\sum_{1}^{\infty}\left(a_{n} \cos n x+b_{n} \sin n x\right) \equiv \sum_{0}^{\infty} A_{n}(x) .
$$

We write

$$
\begin{gathered}
\phi(t)=\frac{1}{2}\{f(x+t)+f(x-t)-2 f(x)\} \\
\Phi(t)=\int_{0}^{t}|\phi(u)| d u \text { and } s_{n}(x)=\sum_{0}^{n} A_{k}(x) .
\end{gathered}
$$

Received by the editors October 13, 1983.

1980 Mathematics Subject Classification. Primary 42A24; Secondary 42A20, 40C05.

Key words and phrases. Triangular matrices, Cesàro method, Nörlund method, convergence criteria for Fourier series.

(C) 1984 American Mathematical Society $0002-9939 / 84 \$ 1.00+\$ .25$ per page 
Let

$$
C_{n}(k)=\sum_{m=0}^{k} c_{n, n-m}
$$

and, for $u \geq 0$, define $C_{n}(u)=C_{n}([u])$, where $[u]$ is the greatest integer function.

Throughout the paper $K$ is used to denote an absolute constant, not necessarily the same at each occurrence.

2. We establish the following

THEOREM. Let $\left\{c_{n, k}\right\}$ be nondecreasing with respect to $k$. Let $\chi$ be a positive function defined over $(0, \infty)$ such that as $n \rightarrow \infty$, (i) $n \chi(n)=O(1)$ and (ii) $\int_{1}^{n} \chi(u) C_{n}(u) d u=O(1)$. Then if $\Phi(t)=o(\chi(\pi / t))$, as $t \rightarrow 0+$, the series $\sum A_{n}(x)$ is summable $(C)$ to $f(x)$.

3. Proof. We have that $\left\{c_{n, k}\right\}$ is nonnegative and nondecreasing in $k$. Hence,

$$
(n-k) c_{n, k} \leq \sum_{m=k+1}^{n} c_{n, m} \leq 1 .
$$

Thus for each fixed $k, c_{n, k} \rightarrow 0$ as $n \rightarrow \infty$, that is, $(C)$ is a regular method.

In view of the fact that the convergence of Fourier series at a point is a local property of the generating function, we may take $\phi(t)=0$ over $[\delta, \pi]$, where $0<$ $\delta<\pi$. We choose $\delta$ such that $\Phi(t)=o(\chi(\pi / t))$ for $t \in(0, \delta)$. Let

$$
t_{n}(x)=\sum_{0}^{n} c_{n, k} s_{k}(x)
$$

Then we need to show that $t_{n}(x)-f(x)=o(1)$ as $n \rightarrow \infty$. After the Dirichlet integral, for $n>\pi / \delta$,

$$
\begin{aligned}
t_{n}(x)-f(x) & =\sum_{0}^{n} c_{n, k} s_{k}(x)-f(x)=\frac{1}{\pi} \int_{0}^{\delta} \phi(t) L(n, t) d t \\
& =\frac{1}{\pi}\left\{\int_{0}^{\pi / n}+\int_{\pi / n}^{\delta}\right\}=\mathrm{I}_{1}+\mathrm{I}_{2}, \quad \text { say, }
\end{aligned}
$$

where

$$
L(n, t)=\sum_{0}^{n} \frac{c_{n, k} \sin \left(k+\frac{1}{2}\right) t}{\sin \left(\frac{1}{2} t\right)}
$$

As

we get

$$
|L(n, t)| \leq \pi \sum_{0}^{n}\left(k+\frac{1}{2}\right) c_{n, k} \leq \pi\left(n+\frac{1}{2}\right),
$$

$$
\left|\mathrm{I}_{1}\right| \leq\left(n+\frac{1}{2}\right) \int_{0}^{\pi / n}|\phi(t)| d t=o(n \chi(n))=o(1)
$$

as $n \rightarrow \infty$.

Next, in view of the order estimates of McFadden [4, Lemma 5.11],

$$
\left|\sum_{k=a}^{b} c_{n, n-k} e^{i(n-k) t}\right| \leq K C_{n}(\pi / t)
$$


where $0 \leq a \leq b \leq \infty, 0<t \leq \pi$, and $n$ a positive integer, we obtain

$$
\begin{aligned}
\left|\mathrm{I}_{2}\right| & \leq K \int_{\pi / n}^{\delta} \frac{|\phi(t)| C_{n}(\pi / t)}{t} d t \\
& =K \sum_{k=r}^{n-1} \int_{\pi /(k+1)}^{\pi / k} \frac{|\phi(t)| C_{n}(\pi / t)}{t} d t+K \int_{\pi / r}^{\delta} \frac{|\phi(t)| C_{n}(\pi / t)}{t} d t
\end{aligned}
$$

where $r$ is a positive integer such that $\pi / r \leq \delta<\pi /(r+1)$. As

$$
\begin{gathered}
\int_{\pi /(k+1)}^{\pi / k} \frac{|\phi(t)| C_{n}(\pi / t)}{t} d t=\left[\frac{C_{n}(\pi / t)}{t} \Phi(t)\right]_{\pi /(k+1)}^{\pi / k}+\int_{\pi /(k+1)}^{\pi / k} \frac{\Phi(t) C_{n}(\pi / t)}{t^{2}} d t \\
\left|\mathrm{I}_{2}\right| \leq o\left(C_{n}(r)\right)+o\left(n \chi(n) C_{n}(n)\right)+K \int_{\pi / n}^{\delta} \frac{\Phi(t) C_{n}(\pi / t)}{t^{2}} d t \\
=o(1)+o\left(\int_{1}^{n} \chi(u) C_{n}(u) d u\right)=o(1) .
\end{gathered}
$$

This completes the proof of the Theorem.

4. The four corollaries in this section follow as a result of our Theorem.

COROLlaRY 1 (HARDY [2]). Let $\alpha>0$. If $\Phi(t)=o(t)$, as $t \rightarrow 0+$, then $\sum A_{n}(x)$ is summable $(C, \alpha)$ to $f(x)$.

The case $\alpha=1$ is the classical result of Lebesgue (see [10, Theorem III 3.9]).

PROOF. Let $\chi(u)=\pi / u$ and $c_{n, k}=A_{n-k}^{\alpha-1} / A_{n}^{\alpha}$. Then $\chi(\pi / t)=t$ and

$$
C_{n}(u)=\sum_{m=0}^{[u]} c_{n, n-m}=\sum_{m=0}^{[u]} \frac{A_{m}^{\alpha-1}}{A_{n}^{\alpha}}=\frac{A_{[u]}^{\alpha}}{A_{n}^{\alpha}} .
$$

Thus $n \chi(n)=\pi$ and

$$
\int_{1}^{n} \chi(u) C_{n}(u) d u=O\left(n^{-\alpha}\right) \int_{1}^{n} u^{\alpha-1} d u=O(1) \quad \text { as } n \rightarrow \infty .
$$

Hence all the hypotheses of the Theorem are satisfied and the result follows.

Corollary 2. (i) (SIDDIQI [6]). If $\Phi(t)=o(t / \log (2 \pi / t))$, as $t \rightarrow 0+$, then $\sum A_{n}(x)$ is summable $(N, 1 /(n+1))$ to $f(x)$.

(ii) If $\Phi(t)=o(t /\{\log (3 \pi / t) \log \log (3 \pi / t)\})$, as $t \rightarrow 0+$, then $\sum A_{n}(x)$ is summable $(N, 1 /\{(n+2) \log (n+2)\})$.

(iii) If $\Phi(t)=o\left(t /\left\{\log (k \pi / t) \log _{2}(k \pi / t) \cdots \log _{q}(k \pi / t)\right\}\right)$, as $t \rightarrow 0+$, then $\sum A_{n}(x)$ is summable $\left(N, 1 /\left\{(n+k) \log (n+k) \cdots \log _{q-1}(n+k)\right\}\right)$, to $f(x)$, where $\log _{r} x=\log \left(\log _{r-1} x\right)$, for $r \geq 2$, and $k$ is such that $\log _{q} k>0$.

ProOF. To deduce this corollary, note that, in case (i) taking

$$
\chi(u)=\frac{\pi}{u \log 2 u} \quad \text { and } \quad c_{n, k}=\frac{1 /(n+1-k)}{\sum_{0}^{n} 1 /(k+1)},
$$


we obtain

$$
\begin{aligned}
\chi(\pi / t) & =t / \log (2 \pi / t), \\
n \chi(n) & =\pi / \log 2 n=o(1) \quad \text { as } n \rightarrow \infty, \\
C_{n}(u) & =\sum_{0}^{[u]} 1 /(m+1) / \sum_{0}^{n} 1 /(k+1),
\end{aligned}
$$

and thus

$$
\int_{1}^{n} \chi(u) C_{n}(u) d u=O\left(\frac{1}{\log n}\right) \int_{1}^{n} \frac{1}{u} d u=O(1) .
$$

Thus the hypotheses of the Theorem are satisfied and the result follows.

The choice of $\chi, c_{n, k}, C_{n}(u)$, etc., is similarly suggested in each of the cases (ii) and (iii), and the proof of the corollary is completed.

COROLLARY 3. Let $\left\{p_{n}\right\}$ be a nonnegative, nonincreasing sequence and let $p(1 / t)=p([1 / t])$ and $P(1 / t)=P([1 / t])$.

(i) (SINGH [7]). If (a) $\Phi(t)=o(t / \log (\pi / t))$ as $t \rightarrow 0+$, and

(b) $\sum_{1}^{n}\left(P_{k} / k \log (k+1)\right)=O\left(P_{n}\right)$,

then $\sum A_{n}(x)$ is summable $(N, p)$ to $f(x)$.

(ii) (PATI [5]). If (c) $\Phi(t)=o(t / P(1 / t))$ as $t \rightarrow 0+$, and

(d) $\log n=O\left(P_{n}\right)$,

then $\sum A_{n}(x)$ is summable $(N, p)$ to $f(x)$.

(iii) (SINGH [8]). If (e) $\Phi(t)=o(p(1 / t) / P(1 / t))$, as $t \rightarrow 0+$, then $\sum A_{n}(x)$ is summable $(N, p)$ to $f(x)$.

REMARKS. In their theorems both Pati and Singh have assumed an extra hypothesis on $\left\{P_{n}\right\}:$ : $P_{n} \rightarrow \infty$, as $n \rightarrow \infty$ ".

PROOF. Since $\left\{p_{n}\right\}$ is nonnegative and nonincreasing,

$$
(n+1) p_{n} \leq p_{0}+p_{1}+\cdots+p_{n}=P_{n}
$$

Therefore $n p_{n} / P_{n}=O(1)$, as $n \rightarrow \infty$. Taking $c_{n, k}=p_{n-k} / P_{n}$ we obtain

$$
C_{n}(u)=P(u) / P_{n}
$$

Case (i). Take $\chi(u)=1$, for $u \in(0,2)$ and $\chi(u)=\pi /(u \log u)$ for $u \in[2, \infty)$. Then for $t \in(0,1 / 2)$,

$$
\chi(\pi / t)=t / \log (\pi / t)
$$

and, for $n \geq 2$,

$$
n \chi(n)=\pi / \log n .
$$

Thus

$$
n \chi(n)=o(1) \quad \text { as } n \rightarrow \infty \text {. }
$$


Also

$$
\begin{aligned}
\int_{1}^{n} \chi(u) C_{n}(u) d u & =\frac{P_{1}}{P_{n}}+\frac{\pi}{P_{n}} \int_{2}^{n} \frac{P(u)}{u \log u} d u \\
& =\frac{P_{1}}{P_{n}}+\frac{\pi}{P_{n}} \sum_{2}^{n-1} \int_{k}^{k+1} \frac{P(u)}{u \log u} d u \\
& \leq \frac{1}{P_{n}}\left\{P_{1}+\pi \sum_{2}^{n-1} \frac{P_{k}}{k \log k}\right\} \\
& \leq K\left(\frac{1}{P_{n}}\right) \sum_{1}^{n} \frac{P_{k}}{k \log (k+1)} \\
& =O(1) \text { as } n \rightarrow \infty,
\end{aligned}
$$

and the hypotheses of the Theorem are satisfied.

Case (ii). Take $\chi(u)=1 / u P(u)$. Then

$$
n \chi(n)=1 / P(n)=O(1), \quad \text { as } n \rightarrow \infty,
$$

and

$$
\int_{1}^{n} \chi(u) C_{n}(u) d u=\frac{1}{P_{n}} \int_{1}^{n} \frac{1}{u} d u=\frac{\log n}{P_{n}}=O(1) .
$$

Case (iii). Let $\chi(u)=p(u) / P(u)$. Then

$$
n \chi(n)=n p_{n} / P_{n}=O(1),
$$

as shown earlier, and also

$$
\int_{1}^{n} \chi(u) C_{n}(u) d u=\frac{1}{P_{n}} \int_{1}^{n} p(u) d u=O(1) .
$$

Thus in each of these cases, the hypotheses of the Theorem are satisfied and the corollary follows.

COROllary 4 (A CONVERGEnCE CRITERION). Let $\chi$ be a decreasing function such that $\int_{1}^{n} \chi(u) d u=O(1)$. If $\Phi(t)=o(\chi(\pi / t))$, as $t \rightarrow 0+$, then $\sum A_{n}(x)$ converges to $f(x)$.

In particular, if $\chi(\pi / t)$ denotes any of the following:

(i) $t /(\log (2 \pi / t))^{1+\varepsilon}$,

(ii) $t /\left\{\log (k \pi / t)(\log \log (k \pi / t))^{1+\varepsilon}\right\}, \ldots$ where $\varepsilon>0$ and $k$ is appropriately chosen, then $\Phi(t)=O(\chi(\pi / t))$ implies that $\sum A_{n}(x)$ converges to $f(x)$.

REMARKS. This result may be compared with the corresponding classical results on nonconvergence of a Fourier series at a point of continuity, e.g. see $[\mathbf{1 0}$, Theorem VIII 2.4, p. 303]. Thus, in the suggested particular cases, $\varepsilon>0$ may not be replaced by $\varepsilon=0$. For other alternate convergence criteria involving the case $\varepsilon=0$, see $[\mathbf{3}$, Theorems 3, 10; 9, Theorems 2, 3].

We shall need the following result for a proof of Corollary 4 .

LEMMA [1]. Let $\left\{p_{n}\right\}$ satisfy the Kaluza conditions:

$$
\text { for } n \geq 0, \quad p_{n}>0 \text { and } p_{n+1} / p_{n} \leq p_{n+2} / p_{n+1} \leq 1 \text {. }
$$


Then if $\left\{P_{n}\right\}$ is bounded, the method $(N, p)$ is ineffective, i.e. only convergent sequences are summable by the method.

ProOF OF COROLlaRY 4. We first note that as $\chi$ is decreasing,

$$
n \chi(n) \leq \int_{1}^{n} \chi(u) d u=O(1)
$$

Now choosing $c_{n, k}=p_{n-k} / P_{n}$ such that $\left\{p_{n}\right\}$ satisfies the requirements of the Lemma (e.g. $\left\{p_{n}\right\}$ may be taken to be one of the sequences

$$
\left\{\frac{1}{(n+1)(n+2)}\right\}, \quad\left\{\frac{1}{2^{n}}\right\}, \quad\left\{\frac{1}{(n+2)(\log (n+2))^{1+\varepsilon}}, \varepsilon>0\right\},
$$

etc.), we see that the hypotheses of the Theorem are satisfied, and thus we complete the proof.

In the case of the particular instances cited, we note that

$$
\Phi(t)=O\left(t /(\log (2 \pi / t))^{1+\varepsilon}\right), \quad \text { as } t \rightarrow 0+
$$

implies that

$$
\Phi(t)=o\left(t /(\log (2 / t))^{1+2 / \varepsilon}\right), \quad \text { as } t \rightarrow 0+,
$$

and similarly in the other cases, and then the results as claimed follow.

\section{REFERENCES}

1. G. D. Dikshit, A note on Riesz and Nörlund means, Rend. Circ. Mat. Palermo (2) 18 (1969), $49-61$.

2. G. H. Hardy, On the summabritity of Fourier series, Proc. London Math. Soc. (2) 12 (1913), 365-372; also in Collected papers of G. H. Hardy, Vol. 3, Oxford Univ. Press, 1969, pp. 118-125.

3. G. H. Hardy and J. E. Littlewood, Some new convergence criteria for Fourier series, Ann. Scuola Norm. Sup. Pisa (2) 3 (1934), 43-62; also in Collected papers of G. H. Hardy, op. cit., pp. 68-88.

4. L. McFadden, Absolute Nörlund summability, Duke Math. J. 9 (1942), 168-207.

5. T. Pati, A generalisation of a theorem of Iyengar on the harmonic summability of Fourier series, Indian J. Math. 3 (1961), 85-90.

6. J. A. Siddiqi, On the harmonic summabrity of Fourier series, Proc. Nat. Acad. Sci. India Sect. A 28 (1948), 527-531.

7. T. Singh, On Nörlund summability of Fourier series and its conjugate series, Proc. Nat. Inst. Sci. India Part A 29 (1963), 65-73.

8. __ Nörlund summability of Fourier series and its conjugate series, Ann. Mat. Pura Appl. (4) 64 (1964), 123-132.

9. G. Sunouchi, Convergence criteria for Fourier series, Tôhoku Math. J. (2) 4 (1952), 187-193.

10. A. Zygmund, Trigonometric series, Vol. 1, Cambridge Univ. Press, 1968.

Department of Mathematics and Statistics, University of AuCKLAnd, AuCKLAND, NEW ZEALAND 